\title{
Towards accelerated agent-based crowd simulation for Hajj and Umrah
}

\begin{abstract}
There are many scientific applications ranging from weather prediction to oil and gas exploration that requires high-performance computing. It aids industries and researchers to enrich further their advancements. With the advent of general purpose computing over GPUs, most of the applications above are shifting towards High-Performance Computing (HPC). Agent-based crowd simulation is one of the candidates that requires high-performance computing. This type of application is used to predict crowd movement in highly congested areas. One of the most crucial scenarios in which this application can be used is to mimic the movement of the multi-cultural crowd performing Hajj and Umrah in Masjid Al-Haram, Makkah. Adequate performance for an agent-based crowd system is a common problem in computer science. While the existing event planning software, specifically for Hajj and Umrah, are unable to provide the required performance. The main reason is the increasing amount of autonomous pilgrims every year. In this paper, we propose a high performance agent-based crowd simulation that represents pilgrim movement during these rituals. The performance is achieved by parallelizing an open source steering library called OpenSteer using CUDA over GPU. By using our technique, event organizers will be able to simulate large crowds and will also be able to predict whether the developed event plan is viable or not. We have also discussed the architecture and implementation of this parallel Hajj simulation.
\end{abstract}

Keyword: Agent-based; CUDA; GPU; Hajj; OpenSteer; Performance; Simulation 